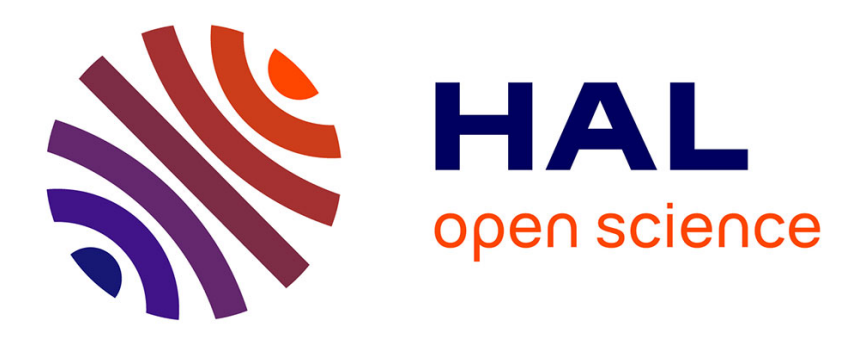

\title{
(e,2e) studies on cadmium autoionizing resonances
}

\author{
N. Martin, D. Thompson, R. Bauman, M. Wilson
}

\section{To cite this version:}

N. Martin, D. Thompson, R. Bauman, M. Wilson. (e,2e) studies on cadmium autoionizing resonances. Journal de Physique IV Proceedings, 1993, 03 (C6), pp.C6-69-C6-78. 10.1051/jp4:1993607 . jpa00251692

\section{HAL Id: jpa-00251692 https://hal.science/jpa-00251692}

Submitted on 1 Jan 1993

HAL is a multi-disciplinary open access archive for the deposit and dissemination of scientific research documents, whether they are published or not. The documents may come from teaching and research institutions in France or abroad, or from public or private research centers.
L'archive ouverte pluridisciplinaire HAL, est destinée au dépôt et à la diffusion de documents scientifiques de niveau recherche, publiés ou non, émanant des établissements d'enseignement et de recherche français ou étrangers, des laboratoires publics ou privés. 


\title{
(e,2e) studies on cadmium autoionizing resonances
}

\author{
N.L.S. MARTIN, D.B. THOMPSON, R.P. BAUMAN and M. WILSON ${ }^{(1)}$ \\ Department of Physics and Astronomy, University of Kentucky, Lexington, KY 40506-0055, U.S.A.
}

\begin{abstract}
We have measured $(e, 2 e)$ energy spectra in cadmium in the $4 d^{9} 5 s^{2} 5 p J=1$ autoionizing region. The incident electron energy was $150 \mathrm{eV}$ and the momentum transfer was $K \approx 0.2$ a.u.. Differences in spectra taken at ejected-electron directions $180^{\circ}$ apart were due to interference terms arising from coherent excitation of $J=1$ and $J=0,2$ autoionizing levels and $J=0,2$ continua. $A b$ initio spectroscopic calculations were carried out which enabled the extraction of relative excitation magnitudes and phases from the experimental data. The results are compared with PWBA calculations.
\end{abstract}

\section{Introduction}

In a coherence experiment an initial state $|i\rangle$ is excited to a final state $|f\rangle$ that may be expressed as a coherent superposition of basis states:

$$
|f\rangle=\sum_{n} a_{n}|n\rangle
$$

where the $a_{n}$ are complex excitation amplitudes. The experiment is designed to detect the existence of interference cross terms of the form $a_{n}^{*} a_{n^{\prime}}$, from which relative magnitudes and phases of the excitation amplitudes can be determined.

The best known experiments on collisionally induced coherence are those on the degenerate magnetic sublevels of He $2{ }^{1} P$ state. ${ }^{1}$ In one version of these experiments, angular distributions of emitted photons, measured in coincidence with electrons scattered from $\mathrm{He}$, yield relative excitation magnitudes and phases for the $L=1$ magnetic sublevels $M_{L}=0,|1|$.

Certain types of $(e, 2 e)$ experiments may also be regarded as coherence experiments where the number of basis states is very large. In coplanar asymmetric

(1) Permanent address: Physics Department, Royal Holloway, University of London, Egham, Surrey TW20 0EX, U.K. 
geometry, if one of the outgoing electrons is fast and the other is slow they may be labelled "scattered" and "ejected" electrons respectively. A typical experiment holds $E_{s c}, \theta_{s c}$, and $E_{e j}$ fixed, while $\theta_{e j}$ is varied. Experiments on direct ionization find strong angular correlations between scattered and ejected electrons: $(e, 2 e)$ ejected-electron angular distributions show two pronounced features, the forward, or binary, peak and the backward, or recoil, peak. If the ejected-electron wavefunction (energy $E=E_{e j}$ ) is expressed in terms of a coherent superposition of angular momentum states $|E \ell m\rangle$ appropriate to the field of the ion, then the angular distribution is of the (simplified) form $\left|\sum_{\ell, m}^{\infty} a_{\ell m} Y_{\ell}^{m}(\theta, \phi)\right|^{2}$. The binary/recoil peak intensity ratio is then a consequence of the angular behaviour of the interference cross-terms $a_{\ell m}^{*} a_{\ell^{\prime} m^{\prime}} Y_{\ell}^{m *} Y_{\ell^{\prime}}^{m^{\prime}}$.

In the limit of small momemtum transfer $K \ll 1$ a.u., the plane wave Born approximation (PWBA) predicts that: (1) with respect to a quantization axis along $\hat{K}$, only states with $m_{\ell}=0$ will be populated, and (2) the phase of the amplitudes ${ }^{2}$ is $\left|\phi_{\ell}\right|=\ell \pi / 2$. For $s$ shell ionization, as $K \rightarrow 0$ the PWBA magnitudes are $\left|a_{\ell 0}\right| \propto K^{\ell-2}$ for $\ell>0$. Thus only a small number of partial waves need be considered. Botero and $\mathrm{Macek}^{3}$ have compared a PWBA and a Coulomb-Born approximation (CBA) calculation of the direct inner shell ionization of carbon. They found that both approximations give similar values for the excitation magnitudes, but that the CBA phases are substantially different from the PWBA result. With a basis set restricted to $\ell \leq 3$ they obtained good agreement between the CBA results and experimental ejected-electron angular distributions. Although the basis set is small, there is still a substantial number of cross-terms involved in the calculated angular distributions, and it is not possible to assess the accuracy of the magnitude and phase of individual terms. Below, we describe $(e, 2 e)$ experiments on Cd involving autoionizing levels, using techniques that we have previously developed. ${ }^{4}$ The analysis of these experiments enables the extraction of the relative magnitudes and ratios of individual cross-terms.

\section{Cadmium Ionization}

Our experiments investigated the process

$$
\mathrm{Cd}\left(5 s^{2}{ }^{1} S_{0}\right)+e_{B} \rightarrow \mathrm{Cd}^{+}\left(5 s^{2} S_{\frac{1}{2}}\right)+e_{e j}+e_{s c}
$$

for an incident electron-beam energy $150 \mathrm{eV}$, scattering angles such that $K \approx 0.2$ a.u., and ejected-electron energies $\approx 2.5 \rightarrow 5 \mathrm{eV}$.

\subsection{Cd calculations}

A description of the ionization process in this ejected-electron energy range requires the inclusion of many autoionizing levels and several continua. To aid in the analysis of the experiments a series of $a b$ initio calculations have been carried out. 
1) HFR CI structure calculations ${ }^{5}$ for level positions and widths of $\mathrm{Cd} 5 p 6 s+$ $5 p 7 s+5 p 8 s+5 p 5 d J=1$ autoionizing levels. (Empirical parameters ${ }^{6}$ were used for $4 d^{9} 5 s^{2} 5 p$.)

2) HFR CI structure calculations for level positions and widths of $\mathrm{Cd} 5 p^{2}+$ $5 p 6 p+5 p 7 p J=0,2$ autoionizing levels.

3) Calculations of distorted wave phase shifts for the continuum electron in $5 s E \ell$ with $E=0.01 \rightarrow 4.0 \mathrm{eV}$ and $\ell=0 \rightarrow 3$. The results at low $E$ are in excellent agreement with experimental $5 s n \ell$ quantum defects. ${ }^{7}$

4) PWBA amplitudes for the excitation of all the above autoionizing levels from the ground state. PWBA direct ionization amplitudes $\mathrm{Cd} 5 s^{2} \rightarrow 5 s E \ell$ for $\ell=0 \rightarrow 6$.

5) The magnitudes and phases of the $a_{J}$ were calculated as a function of energy, using the results of the above calculations in Fano-type theories of autoionization. ${ }^{6,8}$

The autoionizing levels are shown in Table (1). Because of substantial spinorbit interaction, the most appropriate label is the total angular momentum $J$. The levels couple to the appropriate $5 s E \ell$ continua, where $\ell=J$; excitation from the ground state involves $2^{J}$-pole transitions. The ejected-electron spectrum ${ }^{9}$ is dominated by three overlapping dipole-excited $4 d^{9} 5 s^{2} 5 p J=1$ levels of which the broadest is $0.14 \mathrm{eV}$ wide. The levels autoionize into both the singlet and triplet $5 s E p$ continua, resulting in both "parity favoured" and "parity unfavoured" ionization processes. ${ }^{10}$ The 5 pnp $J=0,2$ autoionizing levels may be weakly excited (via monopole and quadrupole transitions) from the small $5 p^{2}$ component of the ground state. ${ }^{11}$ The calculations show that the levels only couple to the singlet continua; thus only parity favoured ionization need be considered.

The PWBA amplitude for direct ionization to $5 s E p$ is negligible, which is in agreement with the experimental evidence. ${ }^{4,12}$ Direct ionization into $5 s E s, E d$ is significant, however, and must be included in the analysis of the experiments. The PWBA amplitudes for higher $\ell$ are sufficiently small that they may be neglected; in what follows, only $J=0,1,2$ continua are included.

\subsection{PWBA $(e, 2 e)$ angular distributions and spectra}

In the PWBA the $(e, 2 e)$ angular distribution of ejected-electrons takes the form

$$
I(\theta) \sim \sum_{J, J^{\prime}} a_{J}^{*} a_{J^{\prime}} P_{J} P_{J^{\prime}}
$$

where angles are measured with respect to the quantization axis $\hat{K}$ and $P_{n}=$ $P_{n}(\cos \theta)$ is a Legendre polynomial. (This expression is a simplified form which omits the possibility of parity unfavoured processes; the analysis of our experiments takes these into account). Since terms where $J+J^{\prime}$ is odd change sign for $\theta \rightarrow \theta+\pi$, 
Table 1: Cadmium Autoionizing Levels. Most of the $J=1$ level energies are known experimentaly. All other levels are $a b$ initio calculated values, adjusted to give a tolerable fit to our data. The $5 p^{2}$ widths are from matrix elements calculated at the $5 p 6 p$ configuration average energy; these differ somewhat from the local values.

\begin{tabular}{|c|c|c|c|c|}
\hline & & & Energy $(\mathrm{eV})$ & Width $(\mathrm{eV})$ \\
\hline \multirow[t]{6}{*}{$J=0$} & $5 p^{2}$ & ${ }^{3} P$ & 0.18 & 0.0007 \\
\hline & & ${ }^{1} S$ & 1.94 & 0.0583 \\
\hline & $5 p 6 p$ & ${ }^{3} P$ & 3.69 & 0.0001 \\
\hline & & ${ }^{1} S$ & 4.12 & 0.0014 \\
\hline & $5 p 7 p$ & ${ }^{3} P$ & 4.49 & 0.0001 \\
\hline & & ${ }^{1} S$ & 4.77 & 0.0005 \\
\hline \multirow[t]{12}{*}{$J=1$} & $4 d^{9} 5 s^{2} 5 p$ & ${ }^{3} P$ & 3.07 & 0.0410 \\
\hline & & ${ }^{1} P$ & 3.81 & 0.1400 \\
\hline & & ${ }^{3} D$ & 3.94 & 0.0030 \\
\hline & $5 p 6 s$ & ${ }^{3} P$ & 2.87 & 0.0538 \\
\hline & & ${ }^{1} P$ & 3.19 & 0.2729 \\
\hline & $5 p 5 d$ & ${ }^{3} D$ & 3.86 & 0.0029 \\
\hline & & ${ }^{3} P$ & 4.03 & 0.0080 \\
\hline & & ${ }^{1} P$ & 4.34 & 0.0003 \\
\hline & $5 p 7 s$ & ${ }^{3} P$ & 4.22 & 0.0270 \\
\hline & & ${ }^{1} P$ & 4.53 & 0.0875 \\
\hline & $5 p 8 s$ & ${ }^{3} P$ & 4.82 & 0.0214 \\
\hline & & ${ }^{1} P$ & 5.07 & 0.0418 \\
\hline \multirow[t]{8}{*}{$J=2$} & $5 p^{2}$ & ${ }^{3} P$ & 0.37 & 0.0194 \\
\hline & & ${ }^{1} D$ & 0.88 & 0.7665 \\
\hline & $5 p 6 p$ & ${ }^{3} D$ & 3.70 & 0.0088 \\
\hline & & ${ }^{3} P$ & 3.98 & 0.0230 \\
\hline & & ${ }^{1} D$ & 4.09 & 0.1300 \\
\hline & $5 p 7 p$ & ${ }^{3} D$ & 4.40 & 0.0247 \\
\hline & & ${ }^{3} P$ & 4.69 & 0.0117 \\
\hline & & ${ }^{1} D$ & 4.79 & 0.0980 \\
\hline
\end{tabular}

and the dipole amplitudes are much greater than the monopole and quadrupole, we may write the following relationships:

$$
I(\theta)+I(\theta+\pi) \sim 2\left|a_{1}\right|^{2} P_{1}^{2} \propto \sigma_{1}(\theta),
$$

where $\sigma_{1}$ is the "photoelectron" cross-section, ${ }^{13}$ and

$$
I(\theta)-I(\theta+\pi) \sim 4\left|a_{1}\right| P_{1} \sum_{J}^{0,2}\left|a_{J}\right| \cos \left(\delta_{1}^{T}-\delta_{J}^{T}\right) P_{J}
$$


Here $\delta_{J}^{T}$ is the total phase ${ }^{4}$ and in both expressions terms involving the product of two small amplitudes have been dropped. Since autoionization is present, both the magnitude and the phase of the amplitudes $a_{J}$ depend strongly with energy across a resonance.

Thus manipulation of pairs of $(e, 2 e)$ energy spectra taken $180^{\circ}$ apart enable different terms to be isolated. The "sum spectrum" strips off the cross-terms to yield the dipole cross-section, whereas the "difference spectrum" reveals the interference cross-terms caused by coherent excitation.

\subsection{Types of $\mathrm{Cd}$ experiments}

We have carried out three different types of experiment in Cd. The experiments enable the determination of the magnitudes and phases of individual cross-terms by making use of the properties of Legendre polynomials.

1) For the "magic angle" between the ejected-electron directions and the momentum-transfer axis, $P_{2}(\hat{k} \cdot \hat{K})$ vanishes. The summed spectrum is thus the total (photoabsorption) cross-section. The difference spectrum contains only $(J=1) \times$ $(J=0)$ interference; the fitted spectrum may be used in the analysis of experiment (2).

2) For ejected-electron directions along the momentum-transfer axis, all the Legendre Polynomials are unity. The summed spectrum is the "parity favoured" partial cross-section ( ${ }^{1} P_{1}$ final state continuum). In the difference spectrum the $(J=1) \times(J=2)$ interference is a maximum; its contribution may be assessed after allowing for the $(J=1) \times(J=0)$ interference component obtained from experiment (1).

3) For ejected-electron directions perpendicular to the momentum-transfer axis, the summed spectrum is the "parity unfavoured" partial cross-section $\left({ }^{3} P_{1}\right.$ final state continuum). The difference spectrum provides a rigorous test of the $a b$ initio calculation result that the $J=0,2$ autoionizing levels couple to the singlet, but not the triplet, continuum; i.e. the interference cross-terms should vanish.

\section{Experimental Apparatus}

The coplanar (e,2e) spectrometer consists of four principal components: an electron gun, a metal vapour atomic beam oven, a scattered electron spectrometer and an ejected electron spectrometer (see Fig 1).

The electron gun is based on a design by Erdman and Zipf, ${ }^{14}$ and is located in a side arm of the main vacuum chamber. This enables the ejected electron spectrometer to be positioned on both sides of the electron beam axis. The metalvapor oven is an ohmically heated cylinder capped by a collimation nozzle $1 \mathrm{~cm}$ long and $1 \mathrm{~mm}$ internal diameter.

The scattered and ejected electron spectrometers are of similar construction. In either case electrons from the interaction region pass through a grounded element 


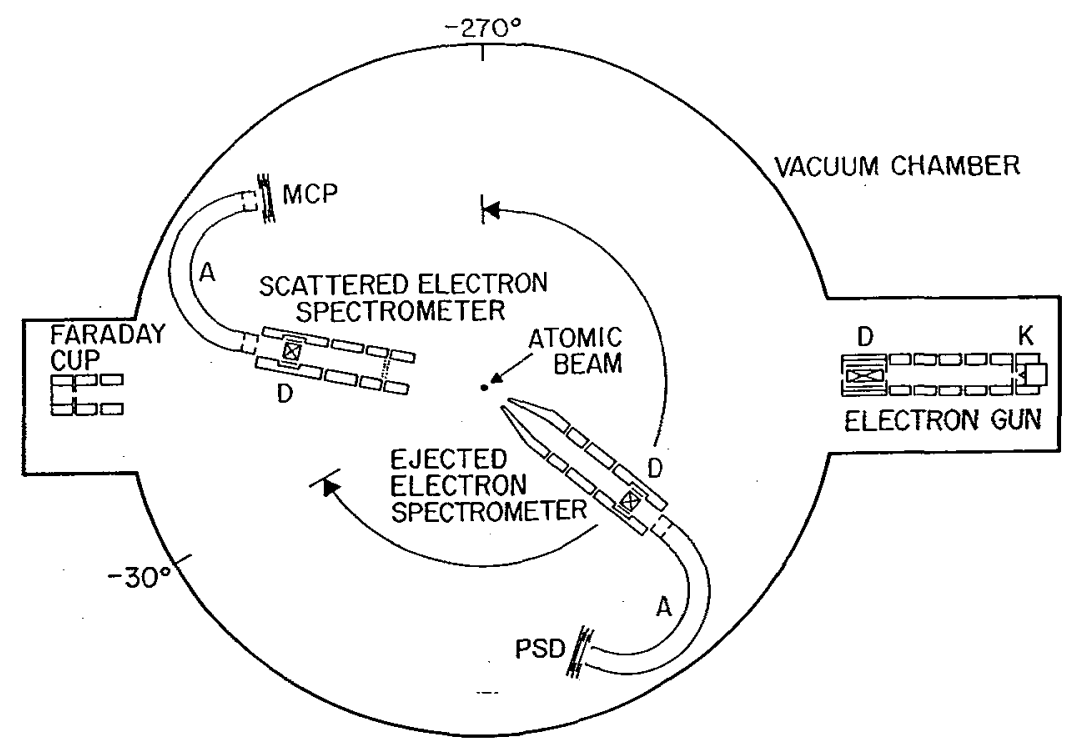

Figure 1. The $(e, 2 e)$ spectrometer. $\mathbf{K}$, cathode; $\mathbf{D}$, deflector; $\mathbf{A}$, hemispherical-sector electrostatic analyser; MCP, microchannel plate electron detector, PSD, position sensitive detector The ejected spectrometer has an angular range $-30^{\circ} \rightarrow-270^{\circ}$, because the electron gun is recessed in a side arm of the vacuum chamber.

and are then transported by a three element lens to the entrance aperture of a hemispherical-sector energy analyser. Electron detection in the scattered channel is by means of a triple microchannel plate (MCP) assembly. The ejected-electron channel incorporates a triple MCP assembly followed by a resistive anode type position sensitive detector (PSD). This enables usable count rates to be maintained at an energy resolution of about $0.04 \mathrm{eV}$. In addition to the pulses output by the resistive anode, which contain positional information, a separate timing pulse is taken from the rear of the second MCP.

The angular range of the scattered spectrometer is $\pm 45^{\circ}$ with respect to the electron beam direction. Because the electron gun is recessed in a side arm, the ejected spectrometer has a useful range of $-30^{\circ} \rightarrow-160^{\circ}$ and $-200^{\circ} \rightarrow-270^{\circ}$, when the scattered spectrometer is at $0^{\circ}$. This range is sufficient to investigate the binary and recoil directions in a single experiment, without recourse to the more usual method of normalizing separate experiments at $\pm \theta_{s c}$.

An internal shield of high magnetic permeability, and external Helmholtz coils, result in an ambient magnetic field of a few milliGauss.

Spectrometer control, data acquisition and analysis is handled by microcom- 
puter. The output strobe from the PSD electronics is used as an interrupt pulse; the position and time information (from a TAC + ADC) is then analysed and recorded by interrupt-driven software. During an experiment, energies and angles are scanned repetitively to minimise the effect of any drift in, for example, the electron beam intensity.

\section{Experimental results}

Since our analysis involves the subtraction of two nearly identical spectra, it is vital to normalize and energy-align the spectra correctly. In all experiments this was achieved by using the non-coincident ejected-electron spectra taken simultaneously with the $(e, 2 e)$ spectra. In the first experiment the ejected-electron directions are $\pm 90^{\circ}$ about the electron-beam axis, and the non-coincident spectra should be identical (to within the smooth secondary-electron background). In the remaining experiments, three Auger peaks ${ }^{9}$ were used; since these correspond to double ionization, for which the direct process is negligible, these have the same intensity for ejected-electron directions $180^{\circ}$ apart. Using these techniques we believe we have aligned the $(e, 2 e)$ spectra to within $1 \mathrm{meV}$ and normalized them to better than $2 \%$.

The energy resolution of the experiments was $\approx 0.04 \mathrm{eV}$. When comparing theory and experiment, the theory was folded with a Gaussian of the appropriate width.

\section{Experiment 1}

In this experiment $(e, 2 e)$ spectra were taken for $\theta_{s c}=+2^{\circ}$ and $\theta_{e j}=-90^{\circ}$ and $-270^{\circ}$. These ejected angles were $54.7^{\circ}$ from the momentum-transfer axis - the "magic angle". The summed spectrum was satisfactorily fitted to the calculated photoabsorbtion cross-section. This enabled a direct comparison of the theoretical and experimental difference spectrum. Because the $J=0$ autoionizing levels are very narrow (see Table 1), we have found that the interference spectrum is determined by two (essentially energy indedendent) parameters, (i) the ratio of the magnitudes of the $5 s \rightarrow E s$ and $4 d \rightarrow 5 p$ excitation matrix elements which provide an overall scale for the difference spectrum, and (ii) the relative phases of these matrix elements which determines the shape of the difference spectrum. The PWBA calculations did not model this spectrum well. By adjusting these two parameters, however, we obtained an excellent fit to the experimental data. We find an empirical value for the matrix element magnitude ratio some 2.4 times the PWBA result. The PWBA relative phase $\phi_{10}=\phi_{1}-\phi_{0}$ is incorrect by an amount given by approximately $\phi_{10}^{\mathrm{EXP}}=\phi_{10}^{\mathrm{PWBA}}-\pi / 4$.

\section{Experiment 2}

For this experiment $(e, 2 e)$ spectra were taken for $\theta_{s c}=+3^{\circ}$ and $\theta_{e j}=-50^{\circ}$ and $-230^{\circ}$. These ejected angles lie along the momentum-transfer axis. The summed 

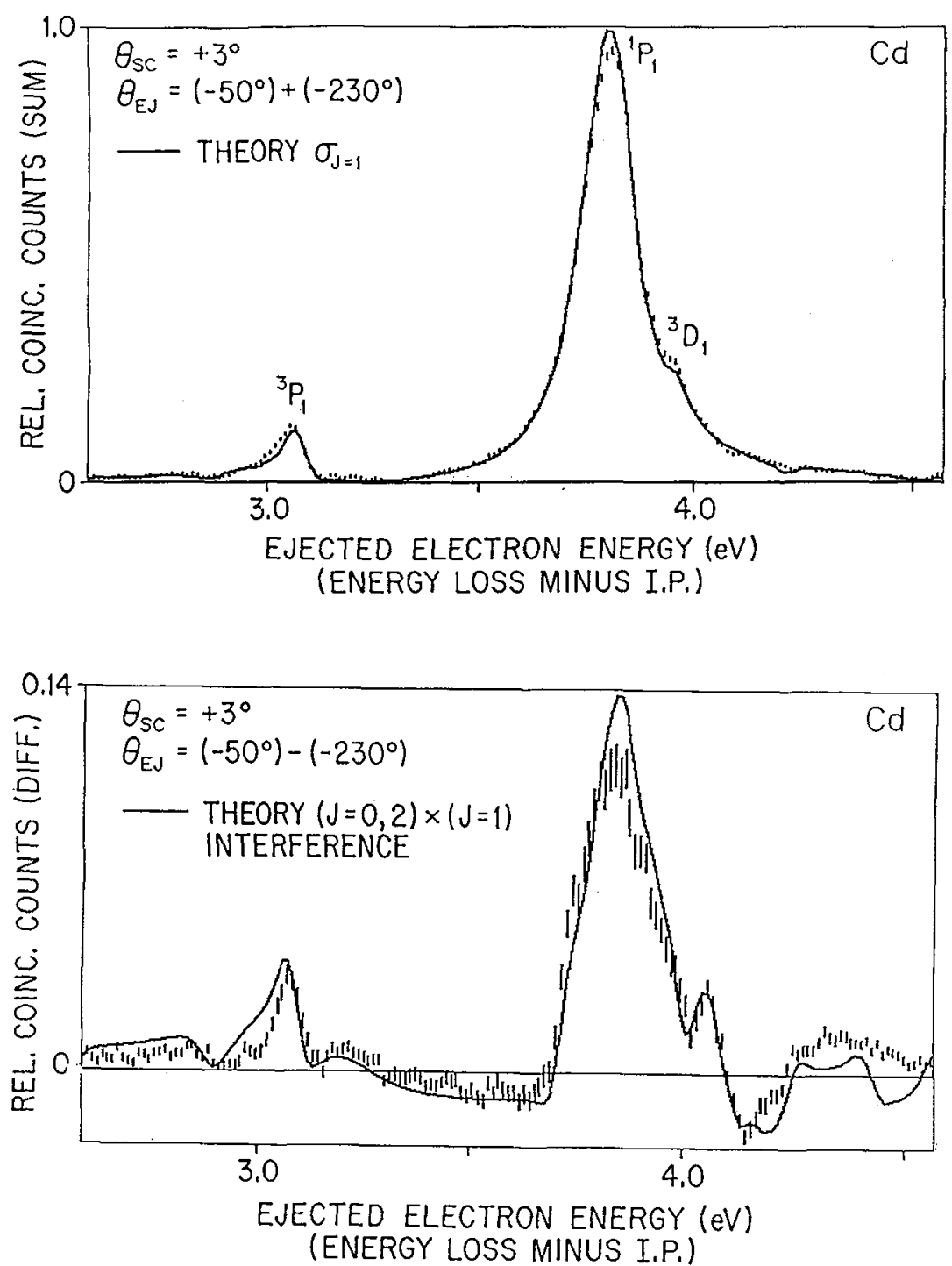

Figure 2. The sum (top) and difference (bottom) spectra from experiment 2. The vertical bars represent the statistical uncertainties. The solid lines are the calculations described in the text.

spectrum (Fig 2, top) was satisfactorily fitted to the calculated photoelectron parityfavoured cross-section. 
The difference spectrum is also shown in Fig 2 (bottom). Note that the maximum of the interference is about $14 \%$ of the cross-section maximum. The theoretical curve uses the $J=1,0$ parameters deduced from experiment 1 and with the $J=1,2$ PWBA parameters adjusted to give a good fit. We find an empirical value for the matrix element magnitude ratio $(5 s \rightarrow E d) /(4 d \rightarrow 5 p)$ which is about twice the PWBA result. The PWBA relative phase is incorrect by an amount given by approximately $\phi_{12}^{\mathrm{EXP}}=\phi_{12}^{\mathrm{PWBA}}+\pi / 4$. Unlike the $J=1,0$ case, the positions and widths of the $5 p n p \quad J=2$ autoionizing levels are important in calculating the difference spectrum. The autoionization amplitudes used in the fit are equal to the PWBA values times the $5 p^{2}$ eigenvector component of the Cd ground state, obtained from a. CI calculation.

\section{Experiment 3}

Lastly, $(e, 2 e)$ spectra were taken for $\theta_{s c}=-2.75^{\circ}$ and $\theta_{e j}=-45^{\circ}$ and $-225^{\circ}$. These ejected angles lie perpendicular to the momentum-transfer axis. The summed spectrum should correspond to the photoelectron parity-unfavoured cross-section, ${ }^{13}$ but, because of the finite angular acceptance of the scattered spectrometer, actually contains a very small contribution from the parity favoured cross-section - a satisfactory fit was obtained by using an effective value $\theta=87^{\circ}$. Within the experimental uncertainty, the difference spectrum was zero. This supports the $a b$ initio result that the $J=2$ autoionizing levels do not couple to the triplet continuum; a finite coupling would have resulted in interference terms between $J=1,2$ parity unfavoured ionization.

\section{Conclusions}

We have obtained relative magnitudes and phases for the excitation amplitudes of coherently excited states of differing total angular momentum $J$. For $J=1$ only autoionization is involved. For $J=0$ direct ionization is the more important process, while for $J=2$, both direct ionization and autoionization are important.

We find that the PWBA predicts relative excitation magnitudes that are incorrect by about a factor of two. The PWBA relative phases also differ from the experimentally obtained values; $\phi_{10}^{\mathrm{EXP}}=\phi_{10}^{\mathrm{PWBA}}-\pi / 4$, and $\phi_{12}^{\mathrm{EXP}}=\phi_{12}^{\mathrm{PWBA}}+\pi / 4$. It is interesting to note that the amount (including signs) of these extra phases are similar to those found in Botero and Macek's ${ }^{3}$ CBA/PWBA calculation of carbon inner shell ionization.

The success of the present experiments depended on the existence of the $J=1$ autoionizing states; the shape of the difference spectra was strongly influenced by the excitation phases, while the intensity was determined by the relative excitation magnitudes. The presence of the Auger peaks in the non-coincident spectra enabled the accurate normalization of the pairs of $(e, 2 e)$ spectra, and the design of the spectrometer ensured that both spectra were taken at exactly the same scattering angle. 
Future work will investigate the behaviour of the relative magnitudes and phases at larger scattering angles and larger momentum transfer.

\section{Acknowledgement}

This work was supported by a grant from the United States Department of Energy, Office of Basic Energy Sciences, Division of Chemical Sciences, Fundamental Interactions Branch, under contract DE-FG05-91ER14214.

\section{References}

1. N. Anderson, J. W. Gallagher and I. V. Hertel, Phys. Rep. 165, 1 (1988).

2. N. F. Mott and H. S. W. Massey, The Theory of Atomic Collisions (Clarendon Press, Oxford, 1965), $3^{\text {rd }}$ ed., p. 22.

3. J. Botero and J. H. Macek, Phys.Rev.A 45, 154 (1992).

4. N.L.S. Martin and D.B. Thompson, Phys.Rev.A 43, 2281 (1991).

5. R. D. Cowan, The Theory of Atomic Structure and Spectra (University of California Press, Berkeley, 1981).

6. N. L. S. Martin, J. Phys. B 23, Number 14, 2223 (1990).

7. C. M. Brown and S. G. Tilford, J. Opt. Soc. Am. 65, 1404 (1975).

8. U. Fano, Phys. Rev. 124, 1866 (1961).

9. V. Pejčev, K. J. Ross, D. Rassi and T. W. Ottley, J. Phys. B 10, 459 (1977).

10. U. Fano and D. Dill, Phys. Rev. A 6, 185 (1972); D. Dill, Phys. Rev. A 7, 1976 (1973).

11. J. E. Hansen, Phys. Rev. A 15, 810 (1977).

12. G. V. Marr and J. M. Austin, Proc. R. Soc. A 310, 137 (1969).

13. N. L. S. Martin and D. B. Thompson, J. Phys. B. 25, 115 (1992).

14. P.W. Erdman and E.C. Zipf, Rev.Sci.Instr. 53, 225 (1982). 